\title{
TRAVELLING WAVES IN NONLINEARLY VISCOELASTIC MEDIA AND SHOCK STRUCTURE IN ELASTIC MEDIA*
}

BY

\author{
STUART S. ANTMAN (University of Maryland) \\ AND \\ REZA MALEK-MADANI (United States Naval Academy)
}

1. Introduction. In this paper we study a class of travelling shear waves in incompressible nonlinearly viscoelastic media with three goals:

i) to present exact solutions for a family of properly invariant equations of nonlinear viscoelasticity,

ii) to study the roles'of nonlinear elastic response and nonlinear viscous response in determining the qualitative behavior of solutions,

iii) to study the behavior of solutions in the limit as the viscous dissipation approaches zero in order to illuminate the shock structure of the system of hyperbolic conservation laws that result from this limit process.

An important way to study a hyperbolic system of the form

$$
\mathbf{U}_{t}=\mathbf{f}(\mathbf{U})_{z}
$$

is to examine its relationship to a parabolic system of the form

$$
\mathbf{U}_{t}=\mathbf{f}(\mathbf{U})_{z}+\mathbf{A} \mathbf{U}_{z z}
$$

where $\mathbf{A}$ is a small constant semi-positive-definite matrix. (Here and throughout this paper subscripts cenote partial derivatives.) The motivation for introducing (1.2) comes from gas dynamics: Equation (1.1) may be regarded as describing a onedimensional flow of an inviscid fluid and Equation (1.2) that of a Newtonian fluid. Within solid mechanics, however, there is no compelling reason to expect to find dissipative mechanisms of a kind inspired by that of classical fluid dynamics.

Our Equations (2.12), (2.13), which describe a special class of motions of a very general family of nonlinearly viscoelastic materials, can be cast in the form

$$
\frac{\partial}{\partial t}\left[\begin{array}{l}
u_{1} \\
u_{2} \\
u_{3} \\
u_{4}
\end{array}\right]=\frac{\partial}{\partial z}\left[\begin{array}{c}
u_{3} \\
u_{4} \\
\mu u_{1}+\nu u_{3 z} \\
\mu u_{z}+\nu u_{4 z}
\end{array}\right]
$$

where $\mu$ and $\nu$ depend on $u_{1}^{2}+u_{2}^{2}, 2\left(u_{1} u_{3 z}+u_{2} u_{4 z}\right), u_{3 z}^{2}+u_{4 z}^{2}$. System (1.3) allows a dependence on $\mathbf{u}_{z z}$ far richer than does (1.2). That (1.3) is parabolic-hyperbolic can

${ }^{*}$ Received January 15, 1987. 
be expected to cause serious analytic difficulties (to which we allude in Section 6). On the other hand, the mathematical structure of (1.3) is special in several ways, as a consequence of its role as a correctly posed, fully nonlinear problem of mechanics.

In Sec. 2, we formulate the governing equations for the special motions of our viscoelastic materials. In Sec. 3, we obtain the equations for travelling waves and determine many of their qualitative properties. In Sec. 4 we study the effect of small viscosity, thereby obtaining viscous shock profiles. Here we show the paramount role played by the elastic response and the surprisingly minor role played by the viscous response. In Sec. 5 we discuss shock admissibility criteria. We comment on questions of stability and existence in Sec. 6. In Sec. 7 we compare our results with those for systems like (1.2).

2. Formulation of the governing equations. Let $\{\mathbf{i}, \mathbf{j}, \mathbf{k}\}$ be a fixed right-handed orthonormal basis for Euclidean three-space. The reference configuration for the body under study is the entire space, a typical material point of which has the form $X \mathbf{i}+Y \mathbf{j}+Z \mathbf{k}$ with $X, Y, Z$ real. We study shearing motions of the space that take this material point to the point $x \mathbf{i}+y \mathbf{j}+z \mathbf{k}$ at time $t$ with

$$
x=X+u(Z, t), \quad y=Y+v(Z, t), \quad z=Z .
$$

We henceforth replace the argument $Z$ of the displacements $u$ and $v$ with $z$.

Relative to the basis $\{\mathbf{i}, \mathbf{j}, \mathbf{k}\}$ the left Cauchy-Green tensor $\mathbf{B}$ and the stretching tensor $\mathbf{D}$ have matrices

$$
\mathbf{B}=\left[\begin{array}{ccc}
1+u_{z}^{2} & u_{z} v_{z} & u_{z} \\
u_{z} v_{z} & 1+v_{z}^{2} & v_{z} \\
u_{z} & v_{z} & 1
\end{array}\right], \quad \mathbf{D}=\frac{1}{2}\left[\begin{array}{ccc}
0 & 0 & u_{z t} \\
0 & 0 & v_{z t} \\
u_{z t} & v_{z t} & 0
\end{array}\right]
$$

Note that $\operatorname{det} \mathbf{B}=1$ so that (2.1) describes an isochoric motion.

We assume that the space is filled with an incompressible isotropic homogeneous viscoelastic material of differential type of complexity 1 . For such a material the Cauchy strees $\mathbf{T}$ is given by a constitutive equation of the form

$$
\mathbf{T}=-p \mathbf{I}+\mathbf{S}(\mathbf{B}, \mathbf{D})
$$

where $p$, the unknown pressure field, is the Lagrange multiplier maintaining the constraint of incompressibility, $\mathbf{I}$ is the identity tensor, and $\mathbf{S}$, the extra stress, is an isotropic tensor function of its two arguments. (Cf. [24, Eq. (35.9)]. S could also depend on $p$ (cf. [1]), but such dependence will not affect our analysis.) A representation theorem of Rivlin and Ericksen [21, §27] (cf. [24, Eq. (13.7)]), shows that $\mathbf{S}$ has the form

$$
\begin{gathered}
\mathbf{S}(\mathbf{B}, \mathbf{D})=\psi_{1} \mathbf{B}+\psi_{2} \mathbf{D}+\psi_{3} \mathbf{B}^{2}+\psi_{4} \mathbf{D}^{2}+\psi_{5}[\mathbf{B D}+\mathbf{D B}]+\psi_{6}\left[\mathbf{B}^{2} \mathbf{D}+\mathbf{D B}^{2}\right] \\
+\psi_{7}\left[\mathbf{B D}^{2}+\mathbf{D}^{2} \mathbf{B}\right]+\psi_{8}\left[\mathbf{B}^{2} \mathbf{D}^{2}+\mathbf{D}^{2} \mathbf{B}^{2}\right]
\end{gathered}
$$

where $\psi_{1}, \ldots, \psi_{8}$ are isotropic scalar functions of $\mathbf{B}$ and $\mathbf{D}$. Since the eigenvalues of D are distinct except when $u_{z t}=0=v_{z t}$ or since the eigenvalues of B are distinct except when $u_{z}=0=v_{z}$, a further result of Rivlin and Ericksen [21, §34] (cf. [24, Eq. (11.22)]) asserts that $\psi_{1}, \ldots, \psi_{8}$ depend on the invariants

$$
\operatorname{tr} \mathbf{B}, \operatorname{tr} \mathbf{B}^{2}, \operatorname{tr} \mathbf{B}^{3}, \operatorname{tr} \mathbf{D}, \operatorname{tr} \mathbf{D}^{2}, \operatorname{tr} \mathbf{D}^{3}, \operatorname{tr}(\mathbf{B D}), \operatorname{tr}\left(\mathbf{B D}^{2}\right), \operatorname{tr}\left(\mathbf{D} \mathbf{B}^{2}\right), \operatorname{tr}\left(\mathbf{B}^{2} \mathbf{D}^{2}\right)
$$


(except possibly when $u_{z}, v_{z}, u_{z t}, v_{z t}$ vanish simultaneously). Here tr denotes trace. When $\mathbf{B}$ and $\mathbf{D}$ are given by (2.2), a lengthy computation shows that all entries of (2.5) depend upon $\boldsymbol{\eta}=\left(\eta_{0}, \eta_{1}, \eta_{2}\right)$ where

$$
\eta_{0}=u_{z}^{2}+v_{z}^{2}, \quad \eta_{1}=2\left(u_{z} u_{z t}+v_{z} v_{z t}\right), \quad \eta_{2}=u_{z t}^{2}+v_{z t}^{2} .
$$

Moreover, a further computation shows that (2.4) yields constitutive equations of the form

$$
\begin{aligned}
\mathbf{i} \cdot(\mathbf{S k}) & =\mu(\boldsymbol{\eta}) u_{z}+\nu(\boldsymbol{\eta}) u_{z t}, \\
\mathbf{j} \cdot(\mathbf{S k}) & =\mu(\boldsymbol{\eta}) v_{z}+\nu(\boldsymbol{\eta}) v_{z t}, \\
\mathbf{k} \cdot(\mathbf{S k}) & =\xi(\boldsymbol{\eta}) .
\end{aligned}
$$

We assume that the scalar functions $\mu, \nu, \xi$ are continuously differentiable.

We assume that the density of the reference configuration is uniform. Since the medium is incompressible, this density is also the density of any deformed configuration. Without loss of generality we take this density to be unity. If the medium is subject to zero body force, then (2.3), (2.7) imply that the equations of motion are

$$
\begin{aligned}
-p_{x}+\left[\mu u_{z}+\nu u_{z t}\right]_{z} & =u_{t t}, \\
-p_{y}+\left[\mu v_{z}+\nu v_{z t}\right]_{z} & =v_{t t}, \\
{[-p+\xi]_{z} } & =0 .
\end{aligned}
$$

Since $u$ and $v$ are independent of $x$ and $y$, Eqs. (2.8) and (2.9) imply that $p$ is affine in $x$ and $y$ while (2.10) implies that $p_{z}$ is independent of $x$ and $y$. Thus $p$ must have the form

$$
p(x, y, z, t)=m(t) x+n(t) y+q(z, t) .
$$

If we assume that the pressure field (at infinity) is independent of $x$ and $y$, then $m=0=n$ and (2.8) and (2.9) reduce to the autonomous system

$$
\begin{aligned}
\left(\mu u_{z}+\mu u_{z t}\right)_{z} & =u_{t t}, \\
\left(\mu v_{z}+\nu v_{z t}\right)_{z} & =v_{t t} .
\end{aligned}
$$

After (2.12) and (2.13) are solved, $q$ can be found from (2.10) to within a function of $t$, which is determined by conditions at infinity.

More generally, we could set

$$
U=u_{z}, \quad V=v_{z} .
$$

Then (2.8), (2.9), (2.11) yield, in place of (2.12), (2.13), the related autonomous system

$$
\begin{aligned}
& {\left[\mu U+\nu U_{t}\right]_{z z}=U_{t t},} \\
& {\left[\mu V+\nu V_{t}\right]_{z z}=V_{t t} .}
\end{aligned}
$$

In this case $m, n$ and the various functions of $t$ that arise from integration are determined by conditions at infinity. Note that the presence of certain body forces would also yield (2.15), (2.16). 
To avoid minor technical difficulties we limit our attention to (2.12), (2.13). Note that this system admits the elegant complex form

$$
\left(\mu w_{z}+\nu w_{z t}\right)_{z}=w_{t t}
$$

for $w=u+i v$, with $\eta_{0}=\left|w_{z}\right|^{2}, \eta_{1}=\left(\left|w_{z}\right|^{2}\right)_{t}, \eta_{2}=\left|w_{z t}\right|^{2}$.

We assume that

$$
\mu(\cdot, 0,0)>0 .
$$

This assumption, which is not critical in our development, is a consequence of the Baker-Ericksen inequality (cf. [23, §53]). We also require that $(2.12),(2.13)$ be parabolic in the sense that the matrix

$$
\left[\begin{array}{ll}
\frac{\partial\left(\mu u_{z}+\nu u_{s t}\right)}{\partial u_{z}} & \frac{\partial\left(\mu u_{z}+\nu u_{z t}\right)}{\partial v_{v t}} \\
\frac{\partial\left(\mu v_{z}+\nu v_{s t}\right)}{\partial u_{z t}} & \frac{\partial\left(\mu u_{z}+\nu v_{z t}\right)}{\partial v_{z t}}
\end{array}\right] \text { is positive-definite. }
$$

This condition, which has thermodynamic significance, ensures that the constitutive equations (2.7) describe a dissipative material.

If we let $\mu_{, 0}=\frac{\partial \mu}{\partial \eta_{0}}, \mu_{, 1}=\frac{\partial \mu}{\partial \eta_{1}}$, etc., then (2.19) is equivalent to

$$
\begin{gathered}
\nu+2\left[\mu_{1,} u_{z}^{2}+\left(\mu_{, 2}+\nu_{, 1}\right) u_{z} u_{z t}+\nu_{, 2} u_{z t}^{2}\right]>0 \\
\nu+2\left[\mu_{, 1} v_{z}^{2}+\left(\mu_{, 2}+\nu_{, 1}\right) v_{z} v_{z t}+\nu_{, 2} v_{z t}^{2}\right]>0 \\
\nu^{2}+2 \nu\left[\mu_{, 1} \eta_{0}+\frac{1}{2}\left(\mu_{, 2}+\nu_{, 1}\right) \eta_{1}+\nu_{, 2} \eta_{2}\right]+4\left(\mu_{, 1} \nu_{, 2}-\mu_{, 2} \nu_{, 1}\right)\left(u_{z} v_{z t}-v_{z} u_{z t}\right)^{2}>0
\end{gathered}
$$

From (2.20) we obtain

$$
\nu+\left[\mu_{.1} \eta_{0}+\frac{1}{2}\left(\mu_{, 2}+\nu_{.1}\right) \eta_{1}+\nu_{.2} \eta_{2}\right]>0
$$

We further require that

$$
u_{z t}\left(\mu u_{z}+\nu u_{z t}\right)+v_{z t}\left(\mu v_{z}+\nu v_{z t}\right) \rightarrow \infty \quad \text { as } \quad u_{z t}^{2}+v_{z t}^{2} \rightarrow \infty .
$$

3. Travelling waves. We seek travelling wave solutions of (2.17) of the form

$$
w(z, t)=\hat{w}\left(\frac{z-c t}{\gamma}\right)
$$

where $c$ (without loss of generality) is a positive constant and $\gamma$ is a positive constant to be assigned later. We denote derivatives of $\hat{w}$ with respect to its argument by primes. Substituting (3.1) into (2.17) we obtain the ordinary differential equation

$$
\left(\mu \hat{w}^{\prime}-c \gamma^{-1} \nu \hat{w}^{\prime \prime}\right)^{\prime}=c^{2} \hat{w}^{\prime \prime}
$$

where the arguments of $\mu$ and $\nu$ are $\gamma^{-2}\left|\hat{w}^{\prime}\right|^{2},-c \gamma^{-3}\left(\left|\hat{w}^{\prime}\right|^{2}\right)^{\prime}, c^{2} \gamma^{-4}\left|\hat{w}^{\prime \prime}\right|^{2}$. We integrate (3.2) once to get

$$
\mu \hat{W}-c \gamma^{-1} \nu \hat{W}^{\prime}=c^{2} \hat{W}+a
$$

where

$$
\hat{w}^{\prime} \equiv \gamma \hat{W}=\gamma \hat{U}+i \gamma \hat{V}
$$

and where the constant of integration $a$ can be taken to be real and nonnegative because our problem is invariant under rotations of the complex $W$-plane. (For 
elastic materials $\nu=0$ and $\mu$ depends only on $\eta_{0}=|W|^{2}$, so that (3.3) reduces to a very uninteresting relation. But elastic materials do possess a very interesting family of standing shear waves. Cf. [2].) From now on we omit the carets over $W, U, V$. Note that the scaling of (3.4) is quite natural, and is consistent with (2.14).

We now study how the phase portrait of (3.3) in the complex $W$ phase plane depends on $a, c$, and the constitutive functions $\mu$ and $\nu$. We first examine the degenerate case in which $a=0$, so that (3.3) is equivalent to

$$
c \nu U^{\prime}=\gamma\left(\mu-c^{2}\right) U, \quad c \nu V^{\prime}=\gamma\left(\mu-c^{2}\right) V .
$$

Note that derivatives of $U$ and $V$ are hidden as arguments of $\mu$ and $\nu$ in (3.5). But (2.19) and (2.23) support a global implicit function theorem to the effect that (3.5) is equivalent to a system in standard form, in which $U^{\prime}, V^{\prime}$ are expressed as functions of $U, V, c, \gamma$. The trajectories of (3.5) lie on rays through the origin of the $W$-plane. The singular points of $(3.5)$ consist of the origin together with those circles of radius $A$ about the origin for which

$$
\mu\left(A^{2}, 0,0\right)=c^{2} .
$$

There can be any number of such circles because the only restriction we have imposed on $\mu(\cdot, 0,0)$ is $(2.18)$.

To find the actual nature of these radial trajectories we observe that (3.5) causes the last term in the left side of (2.21) to vanish. Let $S(c, \gamma)$ be the set of all $\left(U, V, U^{\prime}, V^{\prime}\right) \in$ $\mathbf{R}^{4}$ satisfying (3.5). Then (2.21) implies that

$$
\nu\left\{\nu+2\left[\mu_{, 1} \eta_{0}+\frac{1}{2}\left(\mu_{, 2}+\nu_{, 1}\right)_{1}+\nu_{, 2} \eta_{2}\right]\right\}>0
$$

on $S(c, \gamma)$. Thus $\nu$ cannot vanish on $S(c, \gamma)$. Now (2.20) implies that $\nu(0,0,0)>0$. Since $(0,0,0,0) \in S(c, \gamma)$ and since $\nu$ is continuous, we can conclude that $\nu$ is positive on the connected component of $S(c, \gamma)$ containing $(0,0,0,0)$. That $(3.5)$ can be put into standard form implies that $S(c, \gamma)$ is connected. Thus $\nu$ is positive on $S(c, \gamma)$. From (3.5) (or better yet, from its version in polar coordinates) we then find that trajectories move radially outward where $\mu(\eta)>c^{2}$ and $W \neq 0$ and inward where $\mu(\eta)<c^{2}$ and $W \neq 0$ (as the phase $\gamma^{-1}(z-c t)$ increases). Moreover, (3.5) implies that $\mu(\eta)-c^{2}$ can vanish only on the singular circles defined by (3.6) and possibly at the origin. Thus we have a complete phase portrait of (3.5). A typical example is illustrated in Fig. 3.8.

Now we turn our attention to the general case in which $a>0$. Then (3.3) becomes

$$
c \nu U^{\prime}=\gamma\left(\mu-c^{2}\right) U-\gamma a, \quad c \nu V^{\prime}=\gamma\left(\mu-c^{2}\right) V
$$

from which we obtain

$$
c \nu\left[U V^{\prime}-V U^{\prime}\right]=\gamma a V .
$$

We introduce polar coordinates $R, \Theta$ by

$$
U=R \cos \Theta, \quad V=R \sin \Theta,
$$

in terms of which (3.9) becomes

$$
\begin{aligned}
c \nu R^{\prime} & =\gamma\left(\mu-c^{2}\right) R-\gamma a \cos \Theta, \\
c \nu R \Theta^{\prime} & =\gamma a \sin \Theta
\end{aligned}
$$




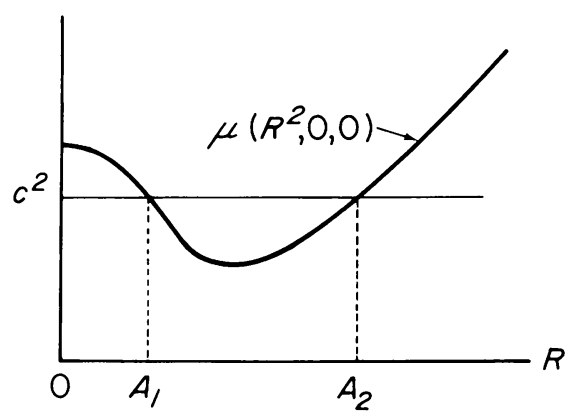

FIG. 3.8a. A typical graph of the constitutive function $\mu(\cdot, 0,0)$ showing the two roots $A_{1}$ and $A_{2}$ of (3.6) when $c^{2}$ has the indicated value. Since $\mu$ is continuously differentiable, the slope of $R \mapsto \mu\left(R^{2}, 0,0\right)$ is 0 at $R=0$.

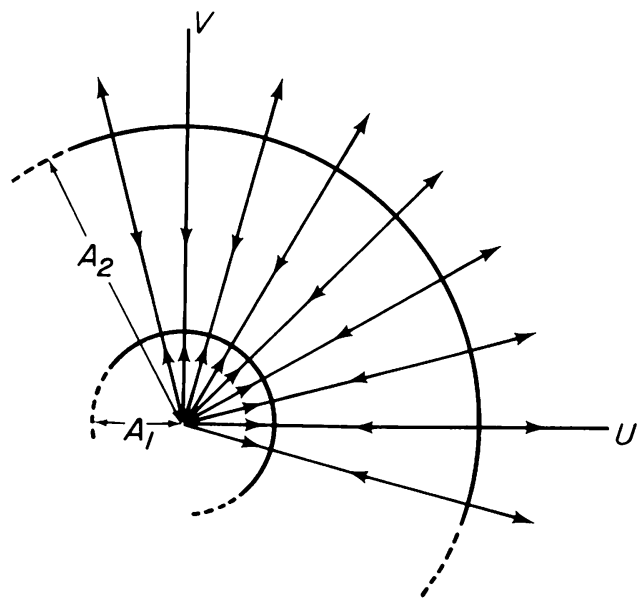

FIG. 3.8b. Phase portrait of (3.5) corresponding to Fig. 3.8a. The two families of trajectories attracted toward the circle of radius $A_{1}$ are heteroclinic. Note that as the $c^{2}$ of Fig. 3.8a is reduced to zero, the two singular circles approach each other, coalesce, and then disappear, whereupon the phase portrait is globally that of a node and contains no heteroclinic trajectories.

where $\eta=\left(R^{2},-2 c \gamma^{-1} R R^{\prime}, c^{2} \gamma^{-2}\left[\left(R^{\prime}\right)^{2}+\left(R \Theta^{\prime}\right)^{2}\right]\right)$.

The singular points of (3.9) occur when $U^{\prime}=0=V^{\prime}$ or possibly where $\nu=0$. In the former case the positivity of $a$ readily shows that the corresponding singular points are of the form $\left(U_{*}, 0\right)$ where $U_{*}$ is a solution of

$$
\left[\mu\left(U^{2}, 0,0\right)-c^{2}\right] U=a .
$$

Suppose $\nu=0$. Then (3.10) implies that $V=0$. Now the assumptions (2.19) and (2.23) support a global implicit function theorem implying that (3.9) can be written in the standard form

$$
U^{\prime}=f(U, V, c, a, \gamma), \quad V^{\prime}=g(U, V, c, a, \gamma) V
$$




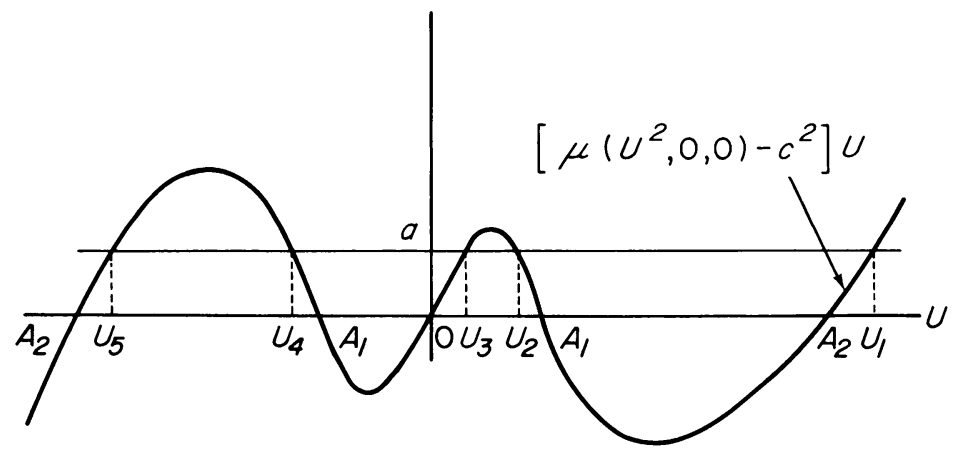

Fig. 3.15. If $\mu(\cdot, 0,0)$ has the form shown in Fig. 3.8a, then $U \mapsto$ $\left[\mu\left(U^{2}, 0,0\right)-c^{2}\right] U$ has the form shown here and the intersection of its graph with a horizontal line with a small enough ordinate $a$ determines the five roots $U_{1}, \ldots, U_{5}$ of $(3.14)$.

Moreover, (3.9) implies that $g$ must be even in $V$. Thus $V^{\prime}=0$ when $V=0$. On the other hand, (2.20b) implies that $\left(V^{\prime}\right)^{2} \nu_{, 2}>0$ when $\nu=0$ (and $V=0$ ), which contradicts the fact that $V^{\prime}=0$. We conclude that not only are there no singular points corresponding to the vanishing of $\nu$, but that $\nu$ can vanish nowhere on solutions of (3.9). Note that the singular points are determined solely by the elastic response. While it would be perfectly reasonable to supplement (2.18) with the requirement that $U \mapsto U \mu\left(U^{2}, 0,0\right)$ be increasing, such an hypothesis would say nothing about the number and disposition of solutions of (3.4). In Fig. 3.15 we illustrate the construction of roots $U_{1}, \ldots, U_{5}$ of $(3.14)$ when $\mu(\cdot, 0,0)$ has the form shown in Fig. 3.8a.

It is important to note that the singular points are collinear. They accordingly correspond to states with constant and parallel $W$ 's. If $U \mapsto U \mu\left(U^{2}, 0,0\right)$ is strictly increasing, then $c^{2}$ and $a$ can always be adjusted so that (3.14) has two prescribed roots.

To classify these singular points, we linearize (3.9) about them, obtaining the uncoupled system

$$
\begin{gathered}
c\left(\nu+2 \mu_{, 1} \gamma^{-3} U_{*}^{2}\right) \delta U^{\prime}=\gamma\left(\mu-c^{2}+2 \mu_{, 0} U_{*}^{2}\right) \delta U, \\
c \nu \delta V^{\prime}=\gamma\left(\mu-c^{2}\right) \delta V
\end{gathered}
$$

for the variations $\delta U, \delta V$. Here $\nu, \mu, \mu_{, 0}, \mu_{, 1}$ have arguments $U_{*}^{2}, 0,0$. Note that (2.20) ensures that the coefficient of $\delta U^{\prime}$ in (3.16a) is positive, while (2.21) ensures that $\nu>0$. The roots of the characteristic equation for (3.16) are

$$
\frac{\gamma\left[\left(\mu-c^{2}\right)+2 \mu_{, 0} U_{*}^{2}\right]}{c\left(\gamma \nu+2 \mu_{, 1} U_{*}^{2}\right)}, \quad \frac{\gamma\left(\mu-c^{2}\right)}{c \nu} .
$$

Note that the numerator of $(3.17 \mathrm{a})$ is just $\gamma$ times the derivative with respect to $U$ of the left side of (3.14) at roots of (3.14). It changes sign at simple roots. Its denominator has fixed sign, by our preceding remarks. From Fig. 3.15, which is typical, we find that $\mu-c^{2}$ is positive at positive roots of (3.14) and negative at 
negative roots. Thus if (3.14) has only simple roots, then the singular points are either saddle points or nodes and their determination follows immediately from (3.14). That (3.16) is uncoupled means that the stable and unstable separatrices through each saddle point are parallel to the coordinate axes at the singular point and that if the two roots in (3.17) are not equal, then each node has axes parallel to the coordinate axes. Thus, we have

THEOREM 3.18. The location and the type of the singular points of $(3.9 \mathrm{a}, \mathrm{b})$ depend only on the elastic response $\mu(\cdot, 0,0)$.

When the situation of Fig. 3.15 holds, $U_{1}$ is an unstable node, $U_{2}$ a saddle point, $U_{3}$ an unstable node, $U_{4}$ a stable node, and $U_{5}$ a saddle point. See Fig. 4.6.

4. Viscous shock profiles. In this section we study the behavior of travelling wave solutions of Eqs. (2.12), (2.13) where $\mu$ and $\nu$ are given by

$$
\mu=\bar{\mu}\left(\eta_{0}\right)+\varepsilon \hat{\mu}(\boldsymbol{\eta}), \quad \nu=\varepsilon \hat{\nu}(\boldsymbol{\eta})
$$

where $\varepsilon$ is a small positive number. We note that unlike simpler models of viscosity (cf., for instance, Conley and Smoller [5], T. P. Liu [17], Majda and Pego [20]) the parameter $\varepsilon$ cannot be scaled out of the system of equations (2.12), (2.13), (4.1). Substituting (4.1) into $(3.9 \mathrm{a}, \mathrm{b})$ and introducing the travelling waves (3.1), we obtain

$$
c \varepsilon \gamma^{-1} \hat{\nu} U^{\prime}=\left(\bar{\mu}-c^{2}\right) U+\varepsilon \hat{\mu} U-a, \quad c \varepsilon \gamma^{-1} \hat{\nu} V^{\prime}=\left(\bar{\mu}-c^{2}\right) V+\varepsilon \hat{\mu} V .
$$

Suppose for large values of $\left|\eta_{1}\right|$ and $\left|\eta_{2}\right|$ that $\hat{\mu}$ and $\hat{\nu}$ have the form

$$
\begin{aligned}
& \hat{\mu}(\boldsymbol{\eta})=M_{1}\left(\eta_{0}\right)\left|\eta_{1}\right|^{k}\left[\operatorname{sign}\left(\eta_{1}\right)\right]^{\beta}+M_{2}\left(\eta_{0}\right)\left|\eta_{2}\right|^{1 / 2}+\ldots \\
& \hat{\boldsymbol{\nu}}(\boldsymbol{\eta})=N_{1}\left(\eta_{0}\right)\left|\eta_{1}\right|^{p}+N_{2}\left(\eta_{0}\right)\left|\eta_{2}\right|^{q / 2}+\ldots
\end{aligned}
$$

where $M_{1}, M_{2}, N_{1}, N_{2}$, are prescribed functions, where $\beta=0$ or 1 , where $k, l, p$, $q>0$, and where the ellipses denote lower-order terms. We now set $\gamma=\varepsilon^{\alpha}$ and seek an appropriate choice of $\alpha$. We substitute (4.3) into (4.2) noting that in the resulting equation the only $\varepsilon$ 's that appear have exponents $0,1-\alpha(1+p), 1-\alpha(1+q), 1-\alpha k$, $1-\alpha l$. These are the candidates for the smallest exponent on $\varepsilon$. Let us consider the case in which $q>p, k-1, l-1$; the treatment of all other cases is similar. Then the candidates for the smallest exponent are 0 and $1-\alpha(1+q)$. If they are not equal, then the coefficients of the smaller must vanish, producing an inadmissible degeneracy. We consequently take $\alpha=(1+q)^{-1}$. After some tedious calculation we find that the leading terms of (3.9), corresponding to the limit of $\varepsilon$ approaching zero, can be reduced to the form

$$
U^{\prime}=\frac{\left(\bar{\mu}-c^{2}\right) U-a}{D}, \quad V^{\prime}=\frac{\left(\bar{\mu}-c^{2}\right) V}{D},
$$

with

$$
D=c N_{2}^{\frac{1}{(1+4)}}\left\{\left[\left(\bar{\mu}-c^{2}\right) U-a\right]^{2}+\left[\left(\bar{\mu}-c^{2}\right) V\right]^{2}\right\}^{\frac{q}{2(q+1)}} .
$$

Since the denominators of (4.4) are the same, we conclude that the only effect of the terms $N_{2}$ and $q$ is merely a nonlinear rescaling of the orbits. These observations lead to 


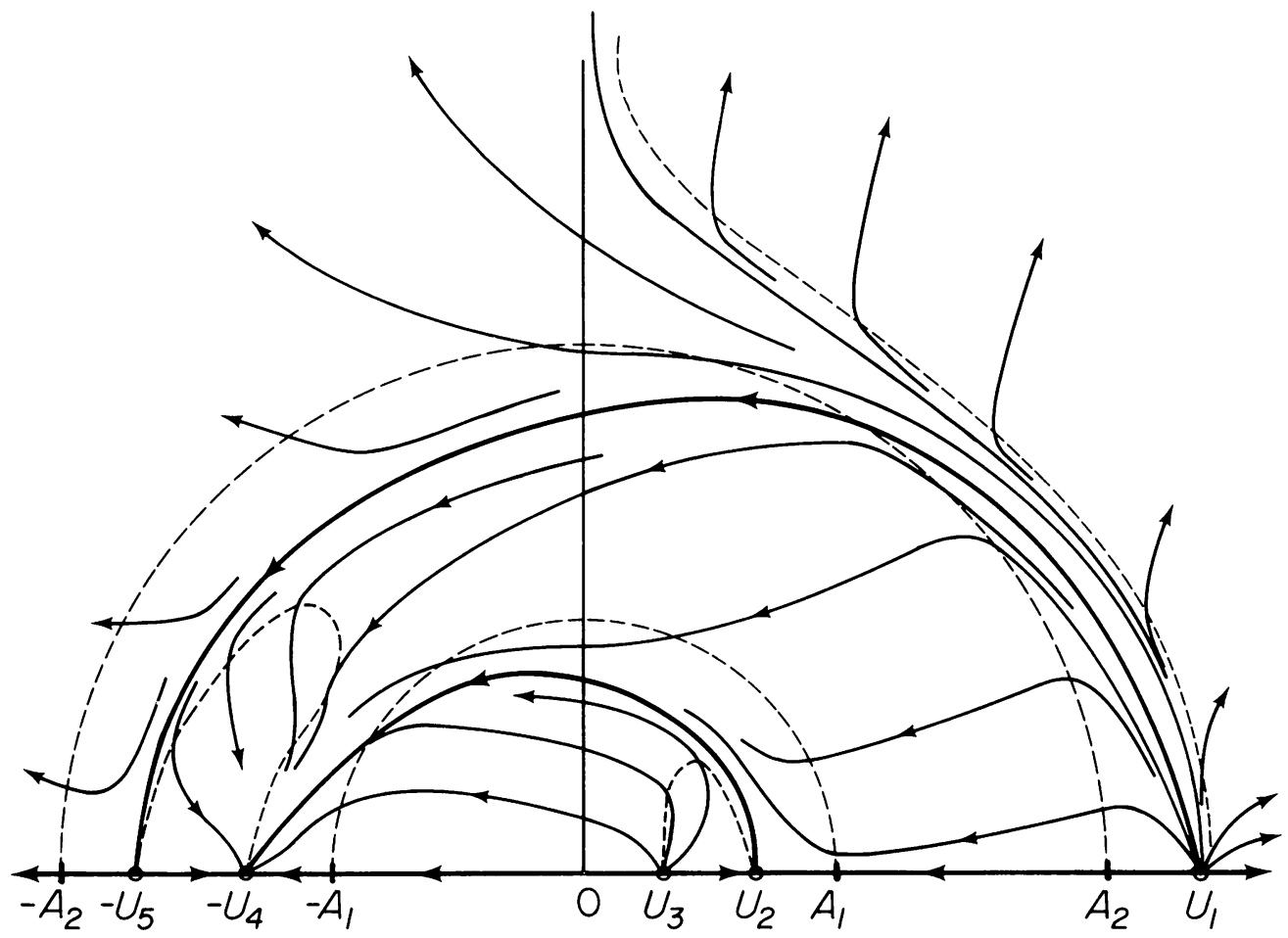

FIG. 4.6. Typical phase portrait for the reduced system (4.4) when $\bar{\mu}$ has the form of $\mu(\cdot, 0,0)$ of Fig. 3.8a. The horizontal and vertical isoclines are shown as dashed curves. Note that the horizontal isoclines other than the $U$-axis are circles. Separatrices are shown as heavy curves. The portrait is symmetric about the $U$-axis. For this problem, the disposition of the horizontal and vertical isoclines completely determines the topological character of the phase portrait.

THEOREM 4.5. The qualitative properties of the phase portrait of (4.4) (which corresponds to $(4.2),(4.3)$ with $\varepsilon=0)$ are completely determined by the elastic response $\bar{\mu}$.

In Fig. 4.6, we exhibit the phase portrait of $(4.4)$ when $\bar{\mu}$ has the form of $\mu(\cdot, 0,0)$ shown in Fig. 3.8a. In this case Fig. 3.15 is valid. It is a straightforward exercise to determine the qualitative behavior of $U$ and $V$ on any trajectory of Fig. 4.6.

In view of the remarks following (3.5) and the construction leading to (4.4), we can write (4.2), (4.3) in the form

$$
\begin{aligned}
& U^{\prime}=\left[\left(\bar{\mu}-c^{2}\right) U-a\right] D^{-1}+\phi(U, V, \varepsilon) \equiv f(U, V, \varepsilon), \\
& V^{\prime}=\left(\bar{\mu}-c^{2}\right) V D^{-1}+\psi(U, V, \varepsilon) V \equiv g(U, V, \varepsilon) V
\end{aligned}
$$

where $\phi$ and $\psi$ are continuous, $\phi(U, V, 0)=0=\psi(U, V, 0)$, and $\phi(\cdot, \cdot, \varepsilon)$ and $\psi(\cdot, \cdot, \varepsilon)$ are continuously differentiable. (Cf. (3.15).) $\phi$ and $\psi$ need not be continuously differentiable in $\varepsilon$ because the most important terms excluded from (4.4) could be proportional to $\varepsilon^{\beta}$ with $\beta \in(0,1)$. But we avoid this difficulty simply by introducing a 
new small parameter $\omega=\varepsilon^{\beta}$, (a change of variables, which, with foresight, could have been performed $a b$ initio). Then the resulting composite functions corresponding to $\phi$ and $\psi$ would be continuously differentiable in $U, V, \omega$. Thus, without loss of generality, we take $\phi$ and $\psi$ of (4.7) to be continuously differentiable.

We next study how the location of the horizontal and vertical isoclines of (3.9) depend on $\varepsilon$ for $\varepsilon$ small.

Let us recall the following elementary theory (which does not seem to be readily accessible). Let $h$ be a small positive number and let $\mathbf{R}^{2} \times(-h, h) \ni(\mathbf{x}, \varepsilon) \mapsto f(\mathbf{x}, \varepsilon) \in$ $\mathbf{R}$ be a continuously differentiable function. Let the gradient $\frac{\partial f}{\partial \mathbf{x}}(\mathbf{x}, 0)$ of $f$ with respect to $\mathbf{x}$ at $\varepsilon=0$ satisfy

$$
\frac{\partial f}{\partial \mathbf{x}}(\mathbf{x}, 0) \neq \mathbf{0} \text { if } f(\mathbf{x}, 0)=0 .
$$

Then

$$
f(\mathbf{x}, 0)=0
$$

defines a $C^{1}$-curve in $\mathbf{R}^{2}$, to which we can give the parametric representation $s \mapsto \hat{\mathbf{x}}(s)$ with $\hat{\mathbf{x}}$ continuously differentiable. By definition of a $C^{1}$-curve, the function

$$
s \mapsto \mathbf{n}(s) \equiv \frac{\partial f}{\partial \mathbf{x}}(\hat{\mathbf{x}}(s), 0) /\left|\frac{\partial f}{\partial \mathbf{x}}(\hat{\mathbf{x}}(s), 0)\right|
$$

assigning the unit normal vector to the curve $\hat{\mathbf{x}}$ is well-defined and continuous. Now set

$$
g(s, t, \varepsilon) \equiv f(\hat{\mathbf{x}}(s)+t \mathbf{n}(s), \varepsilon) .
$$

Then (4.9) implies that $g(s, t, 0)=0$, regarded as an equation for $t$ in terms of $s$, has a solution $t=0$. Since

$$
g_{t}(s, 0,0)=\frac{\partial f}{\partial \mathbf{x}}(\hat{\mathbf{x}}(s), 0) \cdot \mathbf{n}(s) \neq 0
$$

by (4.8) and (4.10), the implicit function theorem implies that for $\varepsilon$ sufficiently small the equation $g(s, t, \varepsilon)=0$ has a continuously differentiable solution $t=\hat{t}(s, \varepsilon)$ with $\hat{t}(s, 0)=0$. Thus for small $\varepsilon$, the curve $s \mapsto \hat{\mathbf{x}}(s)+\hat{t}(s, \varepsilon) \mathbf{n}(s)$ is continuously differentiable and approaches the curve $s \mapsto \hat{\mathbf{x}}(s)$ as $\varepsilon \rightarrow 0$, uniformly on compact subsets of $\mathbf{R}^{2}$.

The horizontal isoclines of (4.7) are the curves in the $(U, V)$-phase plane satisfying

$$
g(U, V, \varepsilon) V=0 .
$$

The isocline $V=0$ is independent of $\varepsilon$, so we need only consider other branches of horizontal isoclines, for which $g(U, V, \varepsilon)=0, V \neq 0$. We identify $g(U, V, \varepsilon)$ with $f(\mathbf{x}, \varepsilon)$ of the preceding paragraph. Then (4.9) is equivalent to

$$
\bar{\mu}\left(U^{2}+V^{2}\right)=c^{2}
$$

(which describe circles) while (4.8) yields

$$
\left(U^{2}+V^{2}\right) \bar{\mu}_{.0}\left(U^{2}+V^{2}\right) \neq 0
$$

for $U, V$ satisfying (4.14), $V \neq 0$. Thus, if $\bar{\mu}$ has the form of $\mu(\cdot, 0,0)$ of Fig. 3.8a, then the horizontal isoclines of (4.7) are close to those of (4.4) on compact sets 
when $\varepsilon$ is small. The treatment of the vertical isoclines is analogous. The condition corresponding to $(4.8)$ is

$$
\left(2 \bar{\mu}_{, 0} U^{2}+\bar{\mu}-c^{2}, 2 \bar{\mu} U V\right) \neq(0,0) \quad \text { when } \quad\left[\bar{\mu}\left(U^{2}+V^{2}\right)-c^{2}\right] U=a .
$$

(Here $a>0$ ). Hence we deduce

THEOREM 4.17. On any compact subset of $\{(U, V): V \neq 0\}$, the horizontal and vertical isoclines of (4.7) approach those of (4.4) uniformly as $\varepsilon \rightarrow 0$. If $R \mapsto$ $\bar{\mu}\left(R^{2}\right)-c^{2}$ has only simple zeros, then the horizontal isoclines of (4.7) approach those of (4.4) uniformly as $\varepsilon \rightarrow 0$ on any compact subset of the phase plane. If $U \mapsto\left[\bar{\mu}\left(U^{2}\right)-c^{2}\right] U-a$ has only simple zeros, the same statement applies to the vertical isoclines.

The above result is a theorem on the structural stability of the isoclines of (4.7). A similar theorem can be obtained for those orbits of (4.7) that connect nondegenerate equilibrium points. First, we note that system (4.4) is gradient-like, i.e., there is a real-valued function $K(U, V)$ which increases along nonconstant solutions of (4.4). To see this we note if $D$ were unity, then the right-hand side of (4.4) would have a potential $k(U, V)$. We can then define $K \equiv D^{-1} k$. Now, suppose that $C$ is an orbit connecting two nondegenerate equilibrium points of (4.4), say a saddle point to an attractive node. As shown before, these points are also nondegenerate equilibrium points of (4.7). Let $N$ be a neighborhood of $C$ containing no other equilibrium points of (4.7). It is easy to show that the function $K$ makes (4.7) gradient-like in $N$ if $\varepsilon$ is sufficiently small. We are now in a position to use a theorem of Conley and Smoller [4]. Since $C$ is an orbit connecting two equilibrium points, its Conley index, $h(C)$, is $\overline{0}$ (cf. Smoller [23], p. 453). We note that $C$ is the maximal invariant set of (4.4) in $N$. A consequence of the definition of the Conley index is that $h(\hat{C})=\overline{0}$ where $\hat{C}$ is the maximal invariant set of (4.7) in $N$. Since (4.7) is gradient-like and $h(\hat{C})=\overline{0}$, Theorem 22.33 of [23] implies that there is a connecting orbit for (4.7) in $N$. These remarks lead to

THEOREM 4.18. The orbits of (4.4) connecting nondegenerate equilibrium points with (Conley) index $\overline{0}$ continue as connecting orbits of (4.7) for $\varepsilon$ sufficiently small.

5. Admissibility of shocks and viscous shock profiles. In this section we study the admissibility of shock waves of

$$
u_{t t}-\left(\bar{\mu} u_{z}\right)_{z}=0, \quad v_{t t}-\left(\bar{\mu} v_{z}\right)_{z}=0
$$

where $\bar{\mu}$ depends only on $\eta_{0}$. Under appropriate conditions on $\bar{\mu}$ the above system is hyperbolic. Let $u_{1}=u_{z}, u_{2}=v_{z}, u_{3}=u_{t}, u_{4}=v_{t}$, and $\mathbf{u}=\left(u_{1}, u_{2}, u_{3}, u_{4}\right)$. Then we can write (5.1) as the fourth-order system

$$
\mathbf{u}_{t}=[\mathbf{f}(\mathbf{u})]_{z}=\frac{\partial \mathbf{f}}{\partial \mathbf{u}} \mathbf{u}_{z}
$$

where

$$
\mathbf{f}(\mathbf{u})=\left[\begin{array}{c}
u_{3} \\
u_{4} \\
\bar{\mu} u_{1} \\
\bar{\mu} u_{2}
\end{array}\right], \quad \frac{\partial \mathbf{f}}{\partial \mathbf{u}}(\mathbf{u})=\left[\begin{array}{cccc}
0 & 0 & 1 & 0 \\
0 & 0 & 0 & 1 \\
\bar{\mu}+2 \bar{\mu}_{, 0} u_{1}^{2} & 2 \bar{\mu}_{, 0} u_{1} u_{2} & 0 & 0 \\
2 \bar{\mu}_{, 0} u_{1} u_{2} & \bar{\mu}+2 \bar{\mu}_{, 0} u_{2}^{2} & 0 & 0
\end{array}\right] .
$$


We note that $(5.1)$ is formally the same as the equations governing the planar motion of a nonlinearly elastic string and, in that context, has been studied extensively by Keyfitz and Kranzer [13] and by Shearer [22]. Also, Antman and Guo [2] exhibited standing wave solutions of (5.1) under assumptions on $\bar{\mu}$ that are physically reasonable for shearing motions of incompressible nonlinearly elastic bodies.

System (5.2), (5.3) is hyperbolic for all values of $\eta_{0}=u_{1}^{2}+u_{2}^{2}$ for which $\bar{\mu}_{, 0}$ and $\bar{\mu}+2 \eta_{0} \bar{\mu}_{.0}$ are positive. In particular, the four eigenvalues of $-\frac{\partial \mathbf{f}}{\partial \mathbf{u}}$ are $\pm \sqrt{\bar{\mu}_{, 0}}$ and $\pm \sqrt{\bar{\mu}+2 \eta_{0} \bar{\mu}_{, 0}}$ with the first two linearly degenerate. Moreover, system (5.2), (5.3) fails to be strictly hyperbolic at all points for which $\bar{\mu}_{0}$ is zero.

Solutions to the initial value problem of a quasilinear hyperbolic system generally develop discontinuities or shocks in finite time. For this reason it is necessary to seek solutions of these systems in the wider class of weak solutions where the propagation of these discontinuities is allowed and well posed. Unfortunately, uniqueness is lost in this larger class of admissible solutions. There has been a considerable amount of research into obtaining the proper admissibility criterion for choosing a unique solution. The question of uniqueness has been settled for some systems of conservation laws (cf. DiPerna [8]). These systems have the property, among others, that the characteristic fields are either linearly degenerate or genuinely nonlinear. The question of uniqueness of solutions still remains open for systems, such as ours, that fail to be genuinely nonlinear. Furthermore, there are several admissibility criteria that, either for physical or mathematical reasons, are candidates for the appropriate extra condition needed for the unique determination of a solution to (5.2). In this section we make a comparison of the "generalized entropy condition" (cf. Liu [16]) and the "viscosity" criterion in the context of (5.2).

Let us denote a discontinuity of $(5.1)$ by $\left(s, \mathbf{u}^{-}, \mathbf{u}^{+}\right)$where $s$ is the speed of propagation and where $\mathbf{u}^{-}$and $\mathbf{u}^{+}$are the limits of $\mathbf{u}$ about the discontinuity from the "left", and the "right". Then the Rankine-Hugoniot jump conditions for (5.1) are

$$
\begin{aligned}
& -s\left[u_{1}\right]=\left[u_{3}\right], \\
& -s\left[u_{2}\right]=\left[u_{4}\right], \\
& -s\left[u_{3}\right]=\left[\bar{\mu} u_{1}\right], \\
& -s\left[u_{4}\right]=\left[\bar{\mu} u_{2}\right],
\end{aligned}
$$

where $[w] \equiv w^{+}-w^{-}$. We next eliminate the velocities $u_{3}$ and $u_{4}$ from (5.4) and introduce the polar coordinates $(r, \theta)$ in the $\left(u_{1}, u_{2}\right)$-plane by setting $u_{1}=r \cos \theta$, $u_{2}=r \sin \theta$, where $r^{2}=\eta_{0}$. We then substitute these relations into (5.4) and conclude that (5.1) admits only shocks for which $[\theta]=0$ or $[\mu]=0$. Shocks of the second kind are linearly degenerate. In the remainder of this paper we discuss the question of admissibility of shock waves of the first kind. Note that the shock across which $\theta$ is continuous corresponds to the collinear singular points for travelling waves discussed in the paragraph preceding that containing (3.16). The shock across which $\bar{\mu}$ is continuous corresponds to travelling waves with $a=0$. (Cf. (3.6).)

Before stating our results, we need to introduce the concept of the generalized entropy criterion. We assume that $\left(s, \mathbf{u}^{-}, \mathbf{u}^{+}\right)$satisfies the Rankine-Hugoniot conditions (5.4) and set $s \equiv s\left(\mathbf{u}^{-}, \mathbf{u}^{+}\right)$. Let $\mathbf{u}^{-}$be in $\mathbf{R}^{4}$. Let $S\left(\mathbf{u}^{-}, \mathbf{u}\right)$ be the set of all points in $\mathbf{R}^{4}$ 
that can be connected to $\mathbf{u}^{-}$by a shock, i.e., let $\left(\mathbf{u}^{-}, \mathbf{u}\right)$ satisfy the Rankine-Hugoniot conditions for some $s$. A triple $\left(s, \mathbf{u}^{-}, \mathbf{u}^{+}\right)$is said to be admissible according to the generalized entropy condition of Liu [16] if

$$
s\left(\mathbf{u}^{-}, \mathbf{u}\right) \geq s\left(\mathbf{u}^{-}, \mathbf{u}^{+}\right)
$$

for every $\mathbf{u}$ on $S\left(\mathbf{u}^{-}, \mathbf{u}^{+}\right)$between $\mathbf{u}^{-}$and $\mathbf{u}^{+}$. For (5.1), inequality (5.5) requires that the chord connecting $\left(r^{-}, r^{-} \bar{\mu}\left(\left(r^{-}\right)^{2}\right)\right.$ to $\left(r^{+}, r^{+} \bar{\mu}\left(\left(r^{+}\right)^{2}\right)\right.$ remain above the graph of the function $r \mapsto r \bar{\mu}\left(r^{2}\right)$ for all $r$ between $r^{-}$and $r^{+}$. We remark that (5.5) is reasonable when $r^{-}$and $r^{+}$are of the same sign but it fails to hold, unless $\bar{\mu}$ is a constant function, when $r^{-}$and $r^{+}$are of opposite sign since $r \mapsto \bar{\mu}\left(r^{2}\right)$ is obviously even.

For constitutive functions that are globally genuinely nonlinear it is not difficult to show that inequality (5.5) reduces to the classical shock conditions of Lax [15], which require that an admissible shock is produced by the intersection in forward time of characteristics of the same family. Specifically, a shock $\left(s, \mathbf{u}^{-}, \mathbf{u}^{+}\right)$is said to be admissible according to Lax's criterion if

$$
\begin{gathered}
{\left.\left[\bar{\mu}+2\left(u_{1}^{2}+u_{2}^{2}\right) \bar{\mu}_{, 0}\right]\right|_{\left(u_{1}^{-}, u_{2}^{-}\right)}>s^{2}>\left.\bar{\mu}\right|_{\left(u_{1}^{-}, u_{2}^{-}\right)},} \\
s^{2}>\left.\left[\bar{\mu}+2\left(u_{1}^{2}+u_{2}^{2}\right) \bar{\mu}_{, 0}\right]\right|_{\left(u_{1}^{+}, u_{2}^{+}\right)}>\left.\bar{\mu}\right|_{\left(u_{1}^{+}, u_{2}^{+}\right)} .
\end{gathered}
$$

In the terminology of [15], inequalities (5.6) are equivalent to the definitions of an admissible 4-shock and an admissible 1 -shock when $\bar{\mu}_{, 0}$ is positive. Moreover, although (5.6) is a reasonable assumption when (5.1) is strictly hyperbolic, we do not expect it to hold for every $u_{1}, u_{2}$ between the points $\left(u_{1}^{-}, u_{2}^{-}\right)$and $\left(u_{1}^{+}, u_{2}^{+}\right)$since the constitutive function, $(0, \infty) \ni r \mapsto r \bar{\mu}\left(r^{2}\right)$, is neither convex nor concave.

Does the shock solution obtained from the vanishing viscosity method satisfy (5.5) and (5.6) and vice versa? To what extent does the validity of (5.5) and (5.6) depend on the form of $\mu$ and $\nu$ ? A partial answer to these questions is given by

THEOREM 5.7. Let $\left(s, \mathbf{u}^{-}, \mathbf{u}^{+}\right)$be a shock that satisfies (5.5) with strict inequality for $\mathbf{u} \neq \mathbf{u}^{+}$and also satisfies (5.6). Let $\hat{\mu}$ and $\hat{\nu}$ satisfy (4.3). Then there is a oneparameter family of travelling wave solutions of $(2.12),(2.13)$ that converges to this shock pointwise almost everywhere as $\varepsilon$ approaches zero.

Proof. Without loss of generality, we assume that $u_{2}^{ \pm}=0$. Following the calculations that led to (4.4) and (4.7) we find that $u_{1}(\xi)$ and $u_{2}(\xi)$, with $\xi=\gamma^{-1}(z-s t)$, satisfy

$$
\begin{aligned}
& u_{1}^{\prime}=\frac{\left(\bar{\mu}-c^{2}\right) u_{1}-a}{D}+\phi\left(u_{1}, u_{2}, \varepsilon\right), \\
& u_{2}^{\prime}=\frac{\left(\bar{\mu}-c^{2}\right) u_{2}}{D}+\psi\left(u_{1}, u_{2}, \varepsilon\right) u_{2}
\end{aligned}
$$

where $\phi$ and $\psi$ are as defined in (4.7). The parameter $a$ is chosen such that $\left(u_{1}^{-}, 0\right)$ and $\left(u_{2}^{-}, 0\right)$ are equilibrium points of $(5.8)$. Condition (5.6) guarantees that $\left(u_{1}^{-}, 0\right)$ is a saddle point and that $\left(u_{1}^{+}, 0\right)$ is an attractive node. The strict inequality in (5.5) implies that there are no other equilibrium points between them. It follows from the second equation of (5.8) that the $u_{1}$-axis is invariant under (4.3). Also, linearization 
of $(4.3)$ about $\left(u_{1}^{-}, 0\right)$ reveals that the unstable manifold of this equilibrium point is tangent to the $u_{1}$-axis. Thus the unstable manifold remains in the $u_{1}$-axis and approaches the attractive equilibrium point $\left(u_{1}^{+}, 0\right)$.

We note that if the strict inequality in (5.5) does not hold, then there is at least a third equilibrium point $\left(u^{*}, 0\right)$ with $u_{1}^{+}<u^{*}<u_{1}^{-}$which prevents the unstable manifold from reaching $\left(u_{1}^{+}, 0\right)$. Thus, in this case, although the generalized entropy condition is satisfied for both $\left(s, \mathbf{u}^{-}, \mathbf{u}^{+}\right)$and $\left(s, \mathbf{u}^{-}, \mathbf{u}^{*}\right)$, only the latter state satisfies the viscosity criterion.

6. Comments on stability and existence. In this section we study the stability of the viscous shock profiles described by Theorem 5.7 when $\mu$ and $\nu$ satisfy (4.1). Furthermore, we present a proof of the existence and uniqueness of the solutions to the initial-boundary value problem of $(2.12),(2.13)$ for a class of constitutive functions.

As in the previous section, (2.12), (2.13) subject to (4.1) can be written as the system

$$
\mathbf{u}_{t}=\mathbf{f}(\mathbf{u})_{z}+\varepsilon\left[\mathbf{G}_{1}\left(\mathbf{u}, \mathbf{u}_{z}\right) \mathbf{u}+\mathbf{G}_{2}\left(\mathbf{u}, \mathbf{u}_{z}\right) \mathbf{u}_{z}\right]_{z}
$$

where

$$
\mathbf{f}(\mathbf{u})=\left[\begin{array}{c}
u_{3} \\
u_{4} \\
\bar{\mu} u_{1} \\
\bar{\mu} u_{2}
\end{array}\right], \quad \mathbf{G}_{1}=\left[\begin{array}{cccc}
0 & 0 & 0 & 0 \\
0 & 0 & 0 & 0 \\
\hat{\mu} & 0 & 0 & 0 \\
0 & \hat{\mu} & 0 & 0
\end{array}\right], \quad \mathbf{G}_{2}=\left[\begin{array}{cccc}
0 & 0 & 0 & 0 \\
0 & 0 & 0 & 0 \\
0 & 0 & \hat{\nu} & 0 \\
0 & 0 & 0 & \hat{\nu}
\end{array}\right] .
$$

The eigenvalues of $\mathbf{M} \equiv \frac{\partial \mathbf{f}}{\partial \mathbf{u}}$ are

$$
\lambda_{1}=-\sqrt{\bar{\mu}+2 \eta_{0} \bar{\mu}_{, 0}}, \quad \lambda_{2}=-\sqrt{\bar{\mu}}, \quad \lambda_{3}=\sqrt{\bar{\mu}}, \quad \lambda_{4}=\sqrt{\bar{\mu}+2 \eta_{0} \bar{\mu}_{, 0}} .
$$

Let $\mathbf{l}_{i}$ and $\mathbf{r}_{i}$ denote the left and right eigenvectors of $\mathbf{M}$ normalized so that $\mathbf{l}_{i} \cdot \mathbf{r}_{i}=1$ $\left(\mathbf{l}_{i}\right.$ is the right eigenvector of $\left.\mathbf{M}^{\mathrm{T}}\right)$. Then

$$
\begin{array}{ll}
\mathbf{l}_{i} \cdot \mathbf{M r}_{i}=\frac{\hat{\nu} u_{1}^{2}}{2 \eta_{0} A_{2}^{2}}\left[A_{2}^{2}+\left(\lambda_{i}^{2}-A_{1}\right)^{2}\right] & \text { for } i=1,4, \\
\mathbf{l}_{i} \cdot \mathbf{M r}_{i}=\frac{\hat{\nu} u_{2}^{2}}{2 \eta_{0} A_{2}^{2}}\left[A_{2}^{2}+\left(\lambda_{i}^{2}-A_{1}\right)^{2}\right] & \text { for } i=1,3,
\end{array}
$$

where $A_{i}=\frac{\partial\left(\bar{\mu} u_{i}\right)}{\partial u_{i}}, i=1,2$.

In recent years there has been a substantial amount of research on the stability of travelling wave solutions of systems of the form (6.1) where $\mathbf{G}_{1}=\mathbf{0}$ and $\mathbf{G}_{2}$ does not depend on $\mathbf{u}_{z}$ (cf. Liu [17], [18], Goodman [10], Majda and Pego [20]). Since $\hat{\nu}$ and $\eta_{0}$ are positive, (6.4) implies that condition (3.21) of [17] is satisfied. For these special systems Liu [17] and Goodman [10] also required that

$$
\mathbf{C} \equiv\left(\mathbf{l}_{1}, \mathbf{l}_{2}, \mathbf{l}_{3}, \mathbf{l}_{4}\right)^{\mathrm{T}} \mathbf{G}_{2}(\mathbf{u})\left(\mathbf{r}_{1}, \mathbf{r}_{2}, \mathbf{r}_{3}, \mathbf{r}_{4}\right)
$$

be positive-definite. A lengthy calculation shows that in our case, however,

$$
\mathbf{C}=\frac{\hat{\nu}}{2}\left[\begin{array}{llll}
1 & 0 & 0 & 1 \\
0 & 1 & 1 & 0 \\
0 & 1 & 1 & 0 \\
1 & 0 & 0 & 1
\end{array}\right]
$$


which is a singular matrix of rank 2. We also point out that our model (with $\mathbf{G}_{1}=\mathbf{0}$, $\mathbf{G}_{2}=\mathbf{G}_{2}(\mathbf{u})$ ) satisfies Majda and Pego's [20] definition of linear stability, but does not satisfy their definition of strict stability.

Recently Liu [18] showed that a condition like (6.5) is not needed to prove the stability of viscous shocks for the one-dimensional equations of a compressible heatconducting Navier-Stokes fluid. Presumably his approach could be extended to (6.1) with $\mathbf{G}_{1}=\mathbf{0}$ and $\mathbf{G}_{2}$ independent of $\mathbf{u}_{z}$. We point out that models of viscoelasticity satisfying these conditions were treated by Kanel' [12] and MacCamy [19]. A richer class of physically reasonable dissipative mechanisms, which could ensure the positive-definiteness of (6.5), are discussed in the last paragraph of Sec. 7. All these observations indicate that the stability of viscous shock profiles remains an important open problem for general systems of the form (6.1).

We next turn to the question of existence, uniqueness, and regularity of solutions to (2.12), (2.13) subject to traction-free boundary conditions specified at the two planes $z=0$ and $z=1$. The program we follow is the one introduced by Friedman [9] in the context of linear parabolic differential equations with nonconstant coefficients and applied successfully by Dafermos [6] to an equation of one-dimensional nonlinear viscoelasticity. The idea of the proof of the existence theorem is as follows. Given a parabolic equation having coefficients that are Hölder continuous together with their appropriate derivatives, one proves that the Hölder norm of the solution, together with its derivatives of order up to 2 in $z$ and up to order 1 in $t$, are bounded by the Hölder norm of the boundary data and the forcing term. The analysis leading to these estimates has two stages. The first is a lengthy and meticulous estimation of the Hölder norm of the second derivative of the solution in terms of the Hölder norm of the solution itself and the forcing term. This analysis relies solely on local estimates of the fundamental solution of the parabolic equation. The second stage is an estimate of the Hölder norm of the solution in terms of the boundary data and the forcing term [9, p. 121]. This estimate relies crucially on the maximum principle, a property that our system lacks in general. The local existence theorem in [6] is proved by combining the above a priori estimate with the Leray-Schauder theory. The proof of existence for all time relies on energy estimates and on growth conditions imposed on the nonlinearities that control the elastic and the viscoelastic responses.

Our existence theorem follows the program by Dafermos [6] very closely. We shall point out some of the differences in our model and otherwise refer the reader to [6] for the methodology. We begin with the following assumption. Let $\mathbf{M}$ be the matrix of partial derivatives of $\mu u_{z}+\nu u_{z t}, \mu v_{z}+\nu v_{z t}$ with respect to $u_{z}, v_{z}$. Let $\mathbf{N}$ be the matrix defined in (2.19). Our assumption concerning the elastic and the viscoelastic responses is

$$
|\mathbf{M v}|^{2} \leq(\mathbf{N v}, \mathbf{v}),
$$

for $\mathbf{v} \in \mathbf{R}^{2}$. This assumption is a generalization of (2.2) of Dafermos [6]. (As pointed out in [3] his assumption may not be physically reasonable for bodies suffering compression, but is perfectly satisfactory for the description of shearing motions.)

A careful study of the proofs of the bounds of the fundamental solutions of linear parabolic equations with nonconstant coefficients obtained in [9] reveals that the 
same bounds can be obtained for such parabolic systems. In order to use this information to obtain a priori bounds on the Hölder norm of the solution in terms of Hölder norms of the forcing term and the initial data we need to invoke a maximum principle. For that reason we assume that $\mu$ and $\nu$ are functions of $\eta_{0}$ only and invoke Theorem VII.2.1 of Ladyženskaja et al. [14] which provides the maximum principle for systems whose hignest-order spatial derivatives are uncoupled. A tedious and lengthy manipulation now shows that all of the a priori estimates obtained in [6] also hold for (2.12), (2.13) and that the proof of the existence and uniqueness presented in [6] goes through in our case. We summarize these remarks in

THEOREM 6.8. Let $\mu=\mu\left(\eta_{0}\right), \nu=\nu\left(\eta_{0}\right)$ be twice continuously differentiable. Let $u_{0}, u_{1}, v_{0}, v_{1}$ be twice continuously differentiable functions of $z$ with $u_{i}^{\prime}(0)=u_{i}^{\prime}(1)=$ $v_{i}^{\prime}(0)=v_{i}^{\prime}(1)=0$ for $i=0,1$. Suppose (2.19) and (6.7) hold. Then (2.12), (2.13) has a unique solution in $C^{2+\alpha}([0,1] \times[0, T])$ satisfying the initial condition

$$
u(z, 0)=u_{0}(z), v(z, 0)=v_{0}(z), u_{t}(z, 0)=u_{1}(z), v_{t}(z, 0)=v_{1}(z),
$$

and the boundary conditions

$$
u_{z}(0, t)=v_{z}(0, t)=u_{z}(1, t)=v_{z}(1, t)=0 .
$$

7. Conclusions. Conley and Smoller [5] studied (1.2) finding that the qualitative behavior of the phase portrait for travelling waves depends crucially on the form of A. Indeed, they exhibit A's for which there are no orbits connecting singular points. Majda and Pego [20] studied (1.2) when A depends on u. They showed that the A's for which there are no connecting orbits could be excluded by the imposition of a further requirement they call strict stability.

In Secs. 3 and 4 we showed that the qualitative properties of the phase portrait for (4.2), (4.3) are scarcely affected by the nature of the viscous dissipation. This result stands in marked contrast to that of Conley and Smoller, whose phase portraits, corresponding to a limited class of dissipative mechanisms, are highly sensitive to changes in the mechanism. Whereas some of their equations lack enough connecting orbits, we confront the opposite problem that some pairs of singular points are joined by an infinity of connecting orbits. The chief difference between our model and theirs is that ours comes from a correctly formulated physical theory and theirs does not. Our equations also fail to be strictly stable in the sense of Majda and Pego [20].

In Theorems 4.17 and 4.18 we proved results on the structural stability of our phase portraits. We point out that the concept of structural stability is in a certain sense inappropriate from the viewpoint of the underlying mechanics. Qualitative properties of solutions need not be invariant under all small changes of (2.12), (2.13), but only under changes of $\mu$ and $\nu$, for only these changes are consonant with the representation theorem delivering (2.4).

Admissibility conditions, such as that of Liu [16], which is based on the mathematical question of uniqueness for Riemann's problem, illuminate some of the issues associated with the multiplicity of connecting orbits, but do not resolve them fully. What may be required is a much deeper investigation of admissibility conditions generated by the evanescence of a whole array of dissipative mechanisms including viscosity, heat conduction, and strain-gradient effects (cf. [7], [11].) 
Acknowledgment. We thank T.-P. Liu for helpful comments. The work of Antman reported here was partially supported by NSF Grant No. DMS-8503317. The work of Malek-Madani was supported by a grant from the United States Naval Academy Research Council.

\section{REFERENCES}

[1] S. S. Antman, Material constraints in continuum mechanics, Atti Acc. Naz. Lincei, Rendiconti, Cl. Sci. fis. mat. nat. Ser. VII, 70, 256-264 (1982)

[2] S. S. Antman and Zhong Heng Guo, Large shearing oscillations of incompressible nonlinearly elastic bodies, J. Elasticity 14, 249-262 (1984)

[3] S. S. Antman and R. Malek-Madani, Dissipative mechanisms, in Metastability and incompletely posed problems, edited by S. S. Antman, J. L. Ericksen, D. Kinderlehrer, I. Müller, IMA volumes in Mathematics and its Applications, Vol. 3, Springer-Verlag, 1-16, 1987

[4] C. C. Conley and J. A. Smoller, Shock waves as limits of progressive wave solutions of higher-order equations, II, Comm. Pure Appl. Math. 25, 131-146 (1972)

[5] C. C. Conley and J. A. Smoller, Topological methods in the theory of shock waves, Proc. Symp. Pure Math. 23, 293-302 (1973)

[6] C. Dafermos, The mixed initial-boundary value problem for the equations of nonlinear one-dimensional viscoelasticity, J. Diff. Eqs. 6, 71-86 (1969)

[7] C. M. Dafermos, Global smooth solutions to the initial-boundary value problem for the equations of one-dimensional nonlinear thermoviscoelasticity, SIAM J. Math. Anal. 13, 397-408 (1982)

[8] R. DiPerna, Uniqueness of solutions to hyperbolic conservation laws, Indiana Univ. Math. Journal 28, $137-188$ (1979)

[9] A. Friedman, Partial differential equations of parabolic type, Prentice-Hall, 1964

[10] J. Goodman, Nonlinear asymptotic stability of viscous shock profiles for conservation laws, Arch. Rat. Mech. Anal. 95, 325-344 (1986)

[11] R. Hagan and M. Slemrod, The viscosity-capillarity admissibility criterion for shocks and phase transitions, Arch. Rat. Mech. Anal. 83, 333-361 (1983)

[12] Ya. I. Kanel', On a model system of equations of one-dimensional gas motion, (in Russian), Diff. Urav. 4, 721-734. Engl. Trans., Diff. Eqs. 4, 374-380 (1968)

[13] B. Keyfitz and H. Kranzer, A system of non-strictly hyperbolic conservation laws arising in elasticity theory, Arch. Rat. Mech. Anal. 72, 219-241 (1980)

[14] O. A. Ladyženskaja, V. A. Solonnikov, and N. N. Ural'ceva, Linear and quasilinear equations of parabolic type, Translations of Mathematical Monographs 23, Amer. Math. Soc., Providence, R.I., 1968

[15] P. D. Lax, Hyperbolic systems of conservation laws, II, Comm. Pure Appl. Math. 10, 537-566 (1957)

[16] T.-P. Liu, The Riemann problem for general systems of conservation laws, J. Diff. Eqs. 18 218-234 (1975)

[17] T.-P. Liu, Nonlinear stability of shock waves for viscous conservation laws, Memoirs of the AMS 328, 1985

[18] T.-P. Liu, Shock waves for compressible Navier-Stokes equations are stable, Comm. Pure Appl. Math. 39, 565-594 (1986)

[19] R. C. MacCamy, Existence, uniqueness and stability of $u_{t t}=\frac{\partial}{\partial x}\left(\sigma\left(u_{x}\right)+\lambda\left(u_{x}\right) u_{x t}\right)$, Indiana Univ. Math. J. 20, 231-238 (1970)

[20] A. Majda and R. Pego, Stable viscosity matrices for systems of conservation laws, J. Diff. Eqs. 56, 229-262 (1985)

[21] R. S. Rivlin and J. L. Ericksen, Stress-deformation relations for isotropic materials, J. Rat. Mech. Anal. 4, 323-425 (1955)

[22] M. Shearer, The Riemann problem for the planar motion of an elastic string, J. Diff. Eqs. 61, 149-163 (1986)

[23] J. A. Smoller, Shock waves and reaction-diffusion equations, Springer-Verlag, 1983

[24] C. Truesdell and W. Noll, The non-linear field theories of mechanics, Handbuch der Physik III/3, Springer-Verlag, 1965 